\title{
Synergistic inhibition of colon carcinoma cell growth by Hedgehog-Glil inhibitor arsenic trioxide and phosphoinositide 3-kinase inhibitor LY294002
}

OncoTargets and Therapy

17 April 2015

Number of times this article has been viewed

\author{
Xinyi Cai ${ }^{1, *}$ \\ Kun Yu ${ }^{1, *}$ \\ Lijuan Zhang ${ }^{2}$ \\ Yunfeng $\mathrm{Li}^{1}$ \\ Qiang Li' \\ Zhibin Yang' \\ Tao Shen' \\ Lincan Duan ${ }^{3}$ \\ Wei Xiong' \\ Weiya Wang' \\ 'Colorectal Cancer Center, \\ ${ }^{2}$ Department of Pathology, \\ ${ }^{3}$ Department of Thoracic Surgery, \\ The Third Affiliated Hospital of \\ Kunming Medical University, \\ Kunming, People's Republic of China \\ *These authors contributed equally \\ to this work
}

\begin{abstract}
The Hedgehog (Hh) signaling pathway not only plays important roles in embryogenesis and adult tissue homeostasis, but also in tumorigenesis. Aberrant Hh pathway activation has been reported in a variety of malignant tumors including colon carcinoma. Here, we sought to investigate the regulation of the Hh pathway transcription factor Gli1 by arsenic trioxide and phosphoinositide 3-kinase (PI3K) inhibitor LY294002 in colon carcinoma cells. We transfected cells with siGli1 and observed a significant reduction of Gli1 expression in HCT116 and HT29 cells, which was confirmed by quantitative real-time polymerase chain reaction and Western blots. Knocking down endogenous Gli1 reduced colon carcinoma cell viability through inducing cell apoptosis. Similarly, knocking down Gli2 using short interfering RNA impaired colon carcinoma cell growth in vitro. To elucidate the regulation of Gli1 expression, we found that both Gli inhibitor arsenic trioxide and PI3K inhibitor LY294002 significantly reduced Gli1 protein expression and colon carcinoma cell proliferation. Arsenic trioxide treatment also reduced Gli1 downstream target gene expression, such as Bcl2 and CCND1. More importantly, the inhibition of Hedgehog-Gli1 by arsenic trioxide showed synergistic anticancer effect with the PI3K inhibitor LY294002 in colon carcinoma cells. Our findings suggest that the Hh pathway transcription factor Gli1 is involved in the regulation of colon carcinoma cell viability. Inhibition of Hedgehog-Gli1 expression by arsenic trioxide and PI3K inhibitor synergistically reduces colon cancer cell proliferation, indicating that they could be used as an effective anticolon cancer combination therapy.
\end{abstract}

Keywords: colon carcinoma cell, Hedgehog pathway, Gli, PI3K, LY294002, arsenic trioxide

\section{Introduction}

Colorectal cancer is the third leading cause of cancer-related death for both women and men in the United States. Early diagnosis and surgical intervention combined with other treatments, such as chemotherapy or radiation therapy, have resulted in dramatically improved outcome. ${ }^{1}$ However, limited effective strategies are available to treat metastatic colon cancer or tumor recurrence. Further understanding of molecular and cellular mechanisms of colon tumorigenesis, progression, and metastasis is critical for the development of novel therapeutics. The role of signaling pathways and their crosstalk in the establishment and maintenance of colorectal cancer are especially important. ${ }^{2,3}$ Exploring the signaling pathways could provide insights into the discovery of novel small-molecule inhibitors for the treatment of colorectal cancer.
Correspondence: Weiya Wang Colorectal Cancer Center, The Third Affiliated Hospital of Kunming Medical University, 519 Kunzhou Road, Kunming 650II8, People's Republic of China

Tel $+8687 \mid$ I3| $706 \mid 3056$

Fax +86 87| 68I8 1942

Email daliduanlincan@163.com 
The Hedgehog $(\mathrm{Hh})$ signaling pathway is a key pathway in embryonic development, patterning of different organs, adult tissue repair, and tumorigenesis. ${ }^{4-6}$ Recently, dysregulation of the Hh pathway has been reported in a variety of tumors. For example, the Hh signaling pathway is constitutively active in medulloblastoma, basal cell carcinoma, small-cell lung cancer, breast cancer, and pancreatic cancer. ${ }^{7-11}$ Many small-molecule inhibitors targeting the Hh pathway, including GDC-0449/Vismodegib, have been tested in clinical trials to treat basal cell, medulloblastoma, ovarian, pancreatic, or metastatic colon cancers. ${ }^{12}$ Activation of the canonical Hh pathway involves the binding of N-terminal forms of Hh to the membrane receptor patched, which then releases the sevenpass transmembrane protein smoothened (SMO). Once the inhibition is relieved, SMO transduces the signal to zinc-finger transcription factors Gli, which translocate into the nucleus and activate the downstream gene transcription. ${ }^{13}$ Among the three Gli family members, Gli1 results in the activation of the Hh pathway downstream target genes, whereas Gli3 is a repressor of the signaling and Gli2 acts as a repressor or activator depending on specific circumstances. ${ }^{14,15}$

The canonical Hh pathway plays important roles during gastrointestinal development. ${ }^{9}$ Although aberrant activation of the Hh pathway has been reported in the oncogenesis of esophageal, small-cell lung, gastric, and pancreatic cancers, its role in colorectal cancer has not been fully understood. ${ }^{11}$ In this study, we sought to investigate the function and regulation of the Hh pathway transcription factor Gli1 in colon carcinoma cells by genetic and pharmacological manipulation of Glil expression.

\section{Materials and methods \\ Cell culture and reagents}

Human colon carcinoma cell line HCT116 and HT29 were purchased from the American Tissue Culture Collection (Manassas, VA, USA) and maintained at $37^{\circ} \mathrm{C}$ in complete Dulbecco's Modified Eagle's medium/F12 (Gibco, Grand Island, NY, USA) supplemented with 10\% fetal bovine serum in humidified $5 \% \mathrm{CO}_{2}$. The Hh signaling pathway was activated using exogenous recombinant human Sonic Hh (Shh) (R\&D Systems, Minneapolis, MN, USA). The specific inhibitors used in the study were arsenic trioxide and LY294002 (Sigma-Aldrich, St Louis, MO, USA).

\section{Gene expression analysis using quantitative real-time polymerase chain reaction (PCR)}

Total RNA of treated cancer cells was isolated using Trizol reagent (Invitrogen, Carlsbad, CA, USA) according to the manufacturer's instructions and was transcribed into complementary DNA with a Complementary DNA Synthesis Kit (Bio-Rad, Hercules, CA, USA). Quantitative real-time PCR was conducted using SYBR Green Supermix (Bio-Rad) in an Applied Biosystems 7300 real-time PCR system. The primers were purchased from Life Technologies (Grand Island, NY, USA). The messenger RNA (mRNA) expression levels were calculated using the $\Delta \Delta \mathrm{Ct}$ method with glyceraldehyde 3-phosphate dehydrogenase (GAPDH) as an internal control.

\section{Short interfering RNA (siRNA) transfection in colon carcinoma cells}

HCT116 or HT29 cells were seeded in six-well plates. After an overnight incubation, the cells were transfected with $20 \mathrm{nM}$ of either scrambled siRNA or siRNA targeting Gli1 or Gli2 (Dharmacon, Lafayette, CO, USA) using INTERFERin (Polyplus transfection) according to the manufacturer's instruction. The cells were collected 48 hours after transfection and subjected to quantitative real-time PCR or Western blot to examine gene expression.

\section{Western blot analysis}

Total protein $(50 \mu \mathrm{g})$ was electrophoresed through an $8 \%$ sodium dodecyl sulfate polyacrylamide gel electrophoresis gel and transferred to a nitrocellulose membrane using an electrophoretic transfer chamber (Millipore, Darmstadt, Germany). The blots were incubated with primary antibodies overnight at $4^{\circ} \mathrm{C}$ followed by incubation with relevant horseradish-peroxidase-conjugated secondary antibodies for 1 hour at room temperature. The primary antibodies for detecting Gli1, cleaved poly adenosine diphosphate ribose polymerase, phospho-Akt (Ser473), Akt, and GAPDH were purchased from Cell Signaling (Danvers, MA, USA). GAPDH was used as a loading control. The signal was detected using an enhanced chemiluminescence Western blot detection kit (Promega, Madison, WI, USA).

\section{Cell viability assay}

Colon carcinoma cells were seeded in 96-well plates and treated with different concentrations of inhibitors as indicated. Cell viability was measured using the CellTiter-Glo luminescent assay (Promega) based on live cell adenosine triphosphate levels.

\section{Colony formation assay}

For anchorage-dependent colony formation assay, cancer cells were seeded in six-well plates at a density of 500 cells per well and treated with inhibitors after 24 hours. 
Single colonies were cultured for 2 weeks and stained with crystal violet. For anchorage-independent colony formation assay, cancer cells were plated on a $0.5 \%$ soft agar layer at a density of $10^{3}$ cells per well in six-well plates. The number of colonies was counted after 3 weeks.

\section{Statistical analysis}

The results were expressed as mean \pm standard deviation. Student's $t$-test was used for statistical analysis. $P$-values less than 0.05 were considered as statistically significant.

\section{Results}

\section{Glil regulates colon carcinoma cell viability}

In order to determine the function of the Hh pathway transcription factor Gli1 in colon carcinoma cells, we specifically reduced Gli1 gene expression using siRNA in HCT116 and HT29 cells. Two siGli1s decreased Gli1 mRNA expression by $60 \%$ to $80 \%$ compared to control siRNA in HT29 cells (Figure 1A). Gli1 protein expression in HT29 cells was also significantly reduced compared to control cells (Figure 1A). Importantly, decreased Gli1 expression caused a $40 \%$ to $60 \%$ reduction of HT29 cell viability. Western blot analysis showed that siGli1 increased the level of cleaved poly adenosine diphosphate ribose polymerase, indicating that knocking down Gli1 reduced cell viability through inducing apoptosis in HT29 cells (Figure 1C). Similar results were observed in HCT116 colon carcinoma cells, suggesting that Gli1 plays a critical role in colon carcinoma cell viability (Figure 1B).
In canonical Hh signaling pathway, Gli1 is an important transcription factor in regulating the transcription of downstream target genes. Using quantitative real-time PCR, we detected a significant reduction of Bcl2 and CCND1 mRNA expression after Gli1 was knocked down by siRNA in HT29 cells, suggesting that Gli1 is a key modulator in the Hh pathway network (Figure 1D). We then tested whether Gli2 is also involved in the regulation of colon carcinoma cell growth since Gli2 belongs to the Gli family. Quantitative real-time PCR revealed that two siRNAs targeting Gli2 significantly decreased Gli2 mRNA expression in HT29 cells (Figure 2A). In addition, siRNA-induced reduction of Gli2 expression dramatically inhibited HT29 cell growth in vitro. Knocking down both Gli1 and Gli2 by siRNA significantly reduced HT29 cell growth by approximately $85 \%$ (Figure $2 \mathrm{~B}$ ). These data suggested that both Gli1 and Gli2 are involved in colon carcinoma cell growth.

\section{Synergistic inhibition of colon carcinoma cell growth by arsenic trioxide and PI3K inhibitor LY294002}

Arsenic trioxide has been approved by the Food and Drug Administration for the treatment of acute promyelocytic leukemia. Studies showed that arsenic trioxide induces apoptosis at high concentrations in acute promyelocytic leukemia cells. ${ }^{16} \mathrm{Kim}$ et al recently reported that arsenic trioxide is able to block Hh-induced ciliary accumulation of Gli2 and reduce the steady-state level of Gli2 in medulloblastoma cells. ${ }^{17}$ Therefore, we tested whether arsenic trioxide could reduce Gli1/2 expression in colon cancer cells. Quantitative real-time
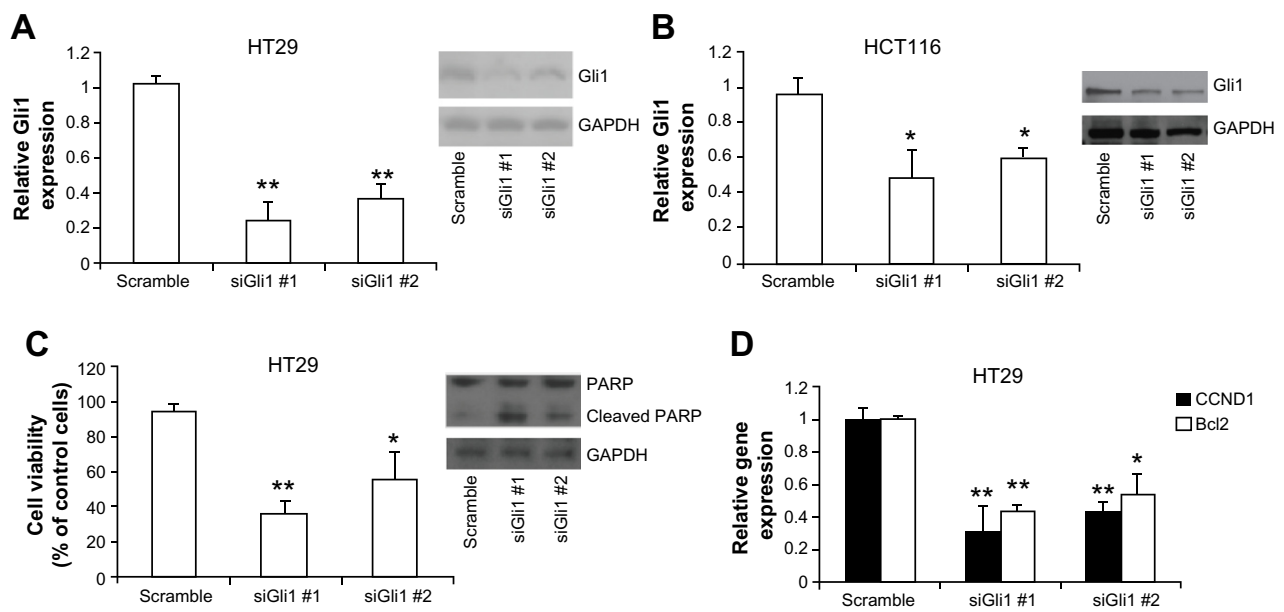

Figure I Knocking down Glil reduces colon carcinoma cell growth.

Notes: (A and B) Glil mRNA expression detected by quantitative real-time PCR and Glil protein expression detected by Western blots in HT29 and HCTII6 cells transfected with scramble siRNA or two different siGlil for 48 hours. (C) Cell viability measured by CellTiter-Glo luminescent assay in HT29 cells transfected with siGlil for 48 hours. Transfection of HT29 cells with siGlil increased the level of cleaved PARP protein. (D) Bcl2 and CCNDI mRNA expression in HT29 cells treated with control or siRNA targeting Glil. $\mathrm{n}=3, * \mathrm{P}<0.05$, $* * \mathrm{p}<0.01$.

Abbreviations: GAPDH, glyceraldehyde 3-phosphate dehydrogenase; mRNA, messenger RNA; PARP, poly adenosine diphosphate ribose polymerase; PCR, polymerase chain reaction; siRNA, short interfering RNA. 
A

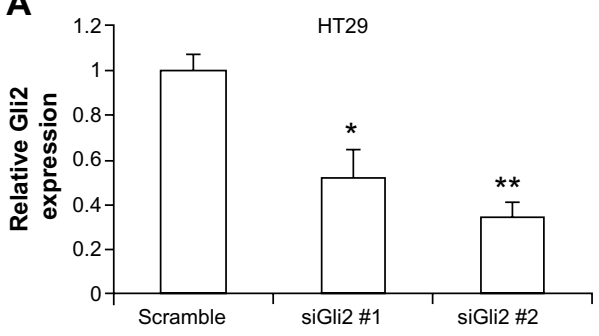

B HT29

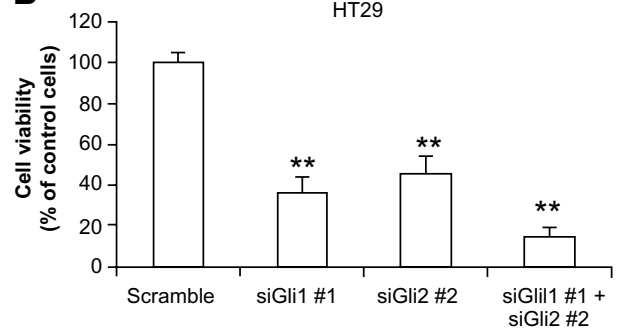

Figure 2 Inhibition of Gli2 reduces HT29 cell growth in vitro.

Notes: (A) Quantitative real-time PCR showed that transfection of HT29 cells with siGli2 for 48 hours decreased Gli2 mRNA expression compared to control cells. (B) CellTiter-Glo luminescent assays revealed that siGli I, siGli2, and the combination inhibited $\mathrm{HT} 29$ cell growth. $\mathrm{n}=3, * \mathrm{P}<0.05, * * P<0.01$.

Abbreviations: mRNA, messenger RNA; PCR, polymerase chain reaction.

PCR showed that $5 \mu \mathrm{M}$ arsenic trioxide dramatically reduced both Gli1 and Gli2 mRNA expression in HT29 cells (Figure 3A and B). We also found that treatment with recombinant human Shh significantly increased both Glil and Gli2 mRNA expression in HT29 cells, which is consistent with previous reports (Figure $3 \mathrm{~A}$ and $\mathrm{B}$ ). Figure $3 \mathrm{C}$ and D show that arsenic trioxide treatment also inhibited Gli1 downstream gene expression, such as CCND1 and Bcl2. Western blot analysis showed that treatment of HT29 cells with 10 $\mu \mathrm{M}$ arsenic trioxide for 48 hours significantly reduced Gli1 protein expression (Figure 4A). Cell viability assay revealed that 3 or $10 \mu \mathrm{M}$ arsenic trioxide treatment significantly decreased HT29 cell growth (Figure 4B). As a positive control, recombinant human Shh promoted HT29 cell proliferation. Moreover, anchorage-dependent and independent colony formation assays showed that $3 \mu \mathrm{M}$ arsenic trioxide significantly reduced the colony forming ability in HT29 cells (Figure 4C and D). The findings suggested that arsenic trioxide not only decreases Gli1 and Gli2 expression, but also impairs colon cancer cell viability and clonogenicity.

We next sought to determine the mechanisms by which Gli1 is tightly regulated in colon carcinoma cells. Since Gli1 is a transcription factor of the Hh pathway, we treated HT29 cells with KAAD cyclopamine (3-Keto$\mathrm{N}$-aminoethyl-N'-aminocaproyldihydrocinnamoyl cyclopamine), a potent inhibitor of SMO, and observed significant growth inhibition compared to control cells (unpublished data). More recently, many reports demonstrated that several signaling pathways crosstalk with the canonical Hh pathway, including transforming growth factor, mitogen-activated protein kinase kinase (MEK)/extracellular signal-regulated kinases (ERK), PI3K/Akt, and nuclear factor kappa-lightchain-enhancer of activated $\mathrm{B}$ cells pathways. ${ }^{18}$ We examined whether Gli1 expression is also regulated by other signaling pathways in colon carcinoma cells using specific kinase inhibitors. Figure 4E and F shows that exposure of HT29 cells to PI3K inhibitor LY294002 at 20 or $40 \mu \mathrm{M}$ significantly reduced cell proliferation and Gli1 protein expression. Since both PI3K inhibitor LY294002 and arsenic trioxide regulate Gli1 protein expression and colon carcinoma cell growth in vitro, we questioned whether the combination of two inhibitors could display a more potent anticancer effect. To address the question, we treated HT29 and HCT116 cells with different doses of arsenic trioxide and LY294002 alone or in combination (different doses of LY294002 in the presence of $1 \mu \mathrm{M}$ arsenic trioxide) for 5 days followed by cell viability
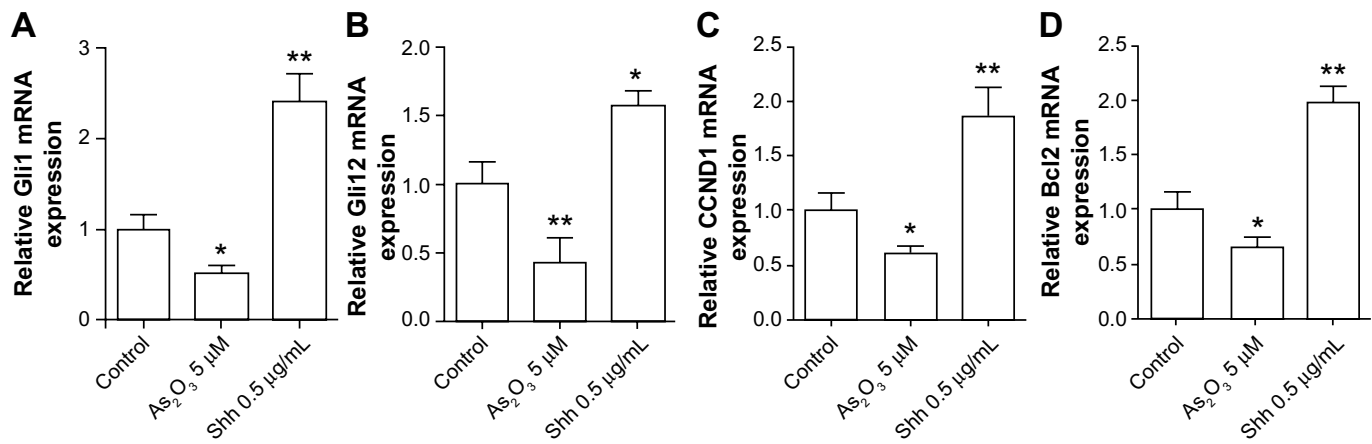

Figure 3 Exposure of HT29 cells to $5 \mu \mathrm{M}$ arsenic trioxide for 48 hours significantly reduced Gli I, Gli2, CCNDI, and Bcl2 mRNA expression.

Notes: Treatment of HT29 cells with $0.5 \mu \mathrm{g} / \mathrm{mL}$ recombinant human Shh significantly increased Gli I, Gli2, CCNDI, and Bcl2 mRNA expression ( $\mathrm{n}=3$, $* P<0.05$, $* * P<0.01$ ). Abbreviations: mRNA, messenger RNA; PCR, polymerase chain reaction; Shh, Sonic Hedgehog. 


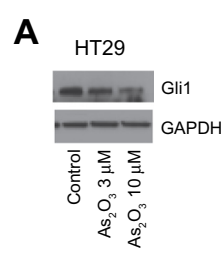

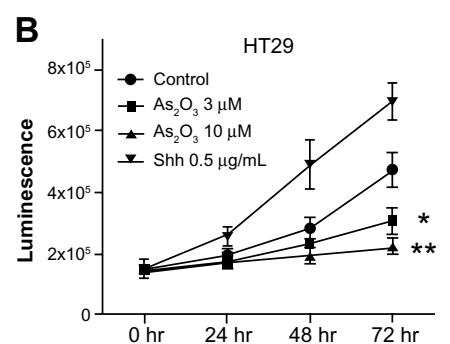

C

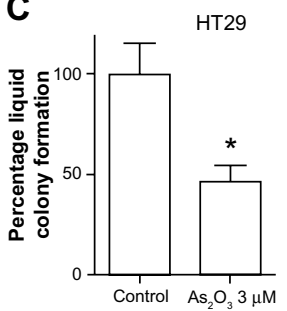

$\mathbf{F}$
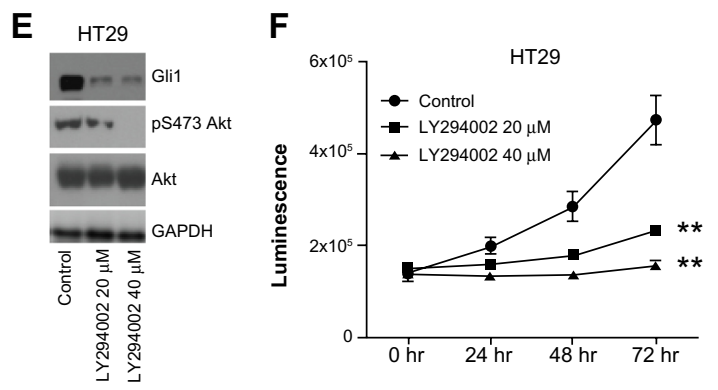

Figure 4 Both arsenic trioxide and PI3K inhibitor LY294002 reduce Glil expression and cell growth in HT29 cells.

Notes: (A and B) Treatment of HT29 cells with 3 or $10 \mu \mathrm{M}$ arsenic trioxide inhibited Glil protein expression and cell growth. Stimulation with $0.5 \mu \mathrm{g} / \mathrm{mL}$ recombinant human Shh enhanced HT29 cell proliferation. (C and D) Exposure of HT29 cells to $3 \mu \mathrm{M}$ arsenic trioxide reduced liquid colony formation and soft agar colony formation. (E and F) Western blots showed that 20 or $40 \mu \mathrm{M}$ LY294002 decreased phospho-Akt level and Glil protein expression. LY294002 treatment also inhibited HT29 cell proliferation $(\mathrm{n}=3, * \mathrm{p}<0.05$, $* * \mathrm{p}<0.0 \mathrm{I})$.

Abbreviations: GAPDH, glyceraldehyde 3-phosphate dehydrogenase; PI3K, phosphoinositide 3-kinase; Shh, Sonic Hedgehog.

assays. We found that $1 \mu \mathrm{M}$ arsenic trioxide significantly reduced the half maximal inhibitory concentration $\left(\mathrm{IC}_{50}\right)$ of LY294002 in both cell lines (Figure 5A and B). Based on the Chou-Talalay model, we calculated the combination index was 0.42 in HT29 cells and 0.48 in HCT116 cells, indicating that blocking the PI3K pathway and arsenic trioxide confer synergism in decreasing colon carcinoma cell growth.

\section{Discussion}

Ectopic activation of the Hh signaling pathway has been identified in a variety of malignant tumors including colorectal cancer. ${ }^{19,20}$ This indicates that the Hh pathway inhibitors are potential therapeutic strategies for colon cancer treatment. In the current study, we found that the
Hh pathway transcription factor Gli1 and Gli2 are critical in controlling colon carcinoma cell proliferation. Besides the Gli inhibitor arsenic trioxide, the PI3K/Akt pathway inhibitor effectively suppresses Gli1 protein expression in colon carcinoma cells. More importantly, blocking PI3K/Akt pathway by LY294002 shows synergistic antitumor activity with arsenic trioxide in colon cancer cells. Our data strongly support that the canonical Hh pathway and PI3K signaling pathway converge to and regulate Gli1 expression and colon carcinoma cell survival.

Until now, there were three major groups of the $\mathrm{Hh}$ pathway inhibitors: Shh neutralizing antibodies, SMO antagonists, and Gli1/2 inhibitors. ${ }^{11,21}$ Cyclopamine, a plantderived steroid, has been demonstrated to have antitumor
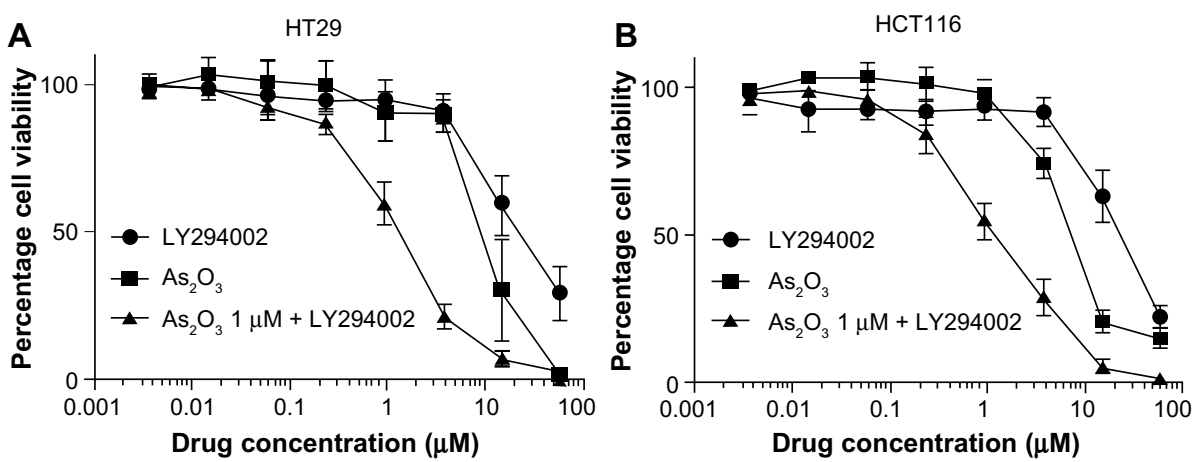

Figure 5 PI3K inhibitor LY294002 and arsenic trioxide synergistically inhibit colon carcinoma cell growth.

Notes: (A and B) HT29 and HCTII6 cells were treated with different doses of LY294002 and arsenic trioxide alone or in combination for 5 days followed by cell viability assay. Abbreviation: PI3K, phosphoinositide 3-kinase. 
activity in preclinical models through binding to and inactivating $\mathrm{SMO} .^{22}$ We found that KAAD cyclopamine significantly decreased colon carcinoma cell proliferation and Gli1 protein expression. With the impressive success of the SMO inhibitor vismodegib in the treatment of basal cell carcinoma, the Hh pathway inhibitors have become novel targeted therapies for cancer. ${ }^{7,23}$ Therefore, many clinical trials using distinct Hh inhibitors alone or in combination with other chemotherapy to treat different malignant tumors including colon cancer have been carried out. ${ }^{12}$ GANT61 and GANT58 are small molecules targeting both Gli1 and Gli2 and effectively block Gli transcriptional activity partially through interfering Gli DNA binding. ${ }^{22}$ We found that arsenic trioxide alone decreased cell viability in both HCT116 and HT29 human colon carcinoma cell lines, and that the combination of arsenic trioxide with PI3K inhibitor LY294002 more potently inhibited cell growth. These findings indicate that directly targeting Gli1 and blocking its upstream regulators together could deliver better anticancer effect in colon carcinoma cells.

Our data and other reports showed a complex Gli1 gene regulatory network in colon carcinoma cells. ${ }^{24}$ Besides canonical Hh pathway components, such as SMO, PTCH, and Sufu, recent findings suggested the crosstalk between the Hh signaling and oncogenic or tumor suppressive signaling is involved in regulating Glil activity. ${ }^{2}$ For example, oncogenic pathways Ras-Raf-MEK or PI3K-Akt-mechanistic-target-ofrapamycin and tumor suppressors phosphatase and tensin homolog or p53 crosstalk with the Hh pathway in certain tumors. ${ }^{18,25-30}$ Since they are key pathways in the regulation of cell proliferation and survival, aberrant activation of these pathways and abnormal Gli1 activity lead to uncontrolled proliferation in human cancers. ${ }^{14}$ Stecca et al reported that endogenous Ras-Raf-MEK and Akt signaling pathways regulate the nuclear translocation and transcriptional activity of Gli1 in melanoma and other cancer cells. ${ }^{31}$ Seto et al showed that mitogen-activated protein kinase signaling pathways regulate Gli activity through a Sufu-independent process in gastric cancer. ${ }^{32}$ In addition, Ji et al demonstrated that oncogenic KRAS is involved in the activation of the $\mathrm{Hh}$ pathway through Raf-MEK-ERK signaling in pancreatic ductal adenocarcinoma cells. ${ }^{25}$ All these studies shed light on the molecular mechanisms by which the Hh pathway mediates carcinogenesis.

Although extensive studies have revealed a lot of details about the mechanisms and functions of the Hh pathway in human cancers, there are still big gaps between the understanding of the Hh pathway and its clinical application.
For example, why are non-canonical Hh pathways activated and involved in Glil regulation in tumors? How is tumor type-specific response determined? What is the mechanism of gene regulation by Gli1 protein in different cancers? Further studies will be needed to address these issues.

In conclusion, data presented in this study defined the inhibitory effects of siGli1 and the Hh-Gli inhibitor arsenic trioxide on colon carcinoma cell viability. We found that Gli1 is regulated by both the canonical Hh pathway and the PI3K/Akt pathway, and that combination therapy with arsenic trioxide and LY294002 had a synergistic therapeutic effect on colon cancer. Our work provides strong evidence for targeting colon carcinoma cells through blocking both PI3K and the Hh pathway.

\section{Acknowledgments}

This study was supported by the Joint Foundation of the Department of Science and Technology of Yunnan Province and Kunming Medical University (number 2012FB062), and the Foundation of the Department of Education of China (number 2012Y025).

\section{Disclosure}

The authors declare no conflicts of interest in this work.

\section{References}

1. Aklilu M, Eng C. The current landscape of locally advanced rectal cancer. Nat Rev Clin Oncol. 2011;8(11):649-659.

2. Bertrand FE, Angus CW, Partis WJ, Sigounas G. Developmental pathways in colon cancer: crosstalk between WNT, BMP, Hedgehog and Notch. Cell Cycle. 2012;11(23):4344-4351.

3. Hanahan D, Weinberg RA. Hallmarks of cancer: the next generation. Cell. 2011;144(5):646-674.

4. Briscoe J, Thérond PP. The mechanisms of Hedgehog signalling and its roles in development and disease. Nat Rev Mol Cell Biol. 2013;14(7): 416-429.

5. Carpenter RL, Lo HW. Hedgehog pathway and GLI1 isoforms in human cancer. Discov Med. 2012;13(69):105-113.

6. Hui CC, Angers S. Gli proteins in development and disease. Annu Rev Cell Dev Biol. 2011;27:513-537.

7. Von Hoff DD, LoRusso PM, Rudin CM, et al. Inhibition of the hedgehog pathway in advanced basal-cell carcinoma. $N$ Engl $J$ Med. 2009;361(12):1164-1172.

8. Teglund S, Toftgård R. Hedgehog beyond medulloblastoma and basal cell carcinoma. Biochim Biophys Acta. 2010;1805(2):181-208.

9. Saqui-Salces M, Merchant JL. Hedgehog signaling and gastrointestinal cancer. Biochim Biophys Acta. 2010;1803(7):786-795.

10. Rudin CM, Hann CL, Laterra J, et al. Treatment of medulloblastoma with hedgehog pathway inhibitor GDC-0449. N Engl J Med. 2009; 361(12):1173-1178.

11. Onishi $\mathrm{H}$, Katano M. Hedgehog signaling pathway as a therapeutic target in various types of cancer. Cancer Sci. 2011;102(10):1756-1760.

12. Xie J, Bartels CM, Barton SW, Gu D. Targeting hedgehog signaling in cancer: research and clinical developments. Onco Targets Ther. 2013;6: $1425-1435$.

13. Varjosalo M, Taipale J. Hedgehog: functions and mechanisms. Genes Dev. 2008;22(18):2454-2472. 
14. Stecca B, Ruiz I Altaba A. Context-dependent regulation of the GLI code in cancer by HEDGEHOG and non-HEDGEHOG signals. J Mol Cell Biol. 2010;2(2):84-95.

15. Ruiz i Altaba A, Mas C, Stecca B. The Gli code: an information nexus regulating cell fate, stemness and cancer. Trends Cell Biol. 2007;17(9): 438-447.

16. Chen GQ, Zhu J, Shi XG, et al. In vitro studies on cellular and molecular mechanisms of arsenic trioxide (As2O3) in the treatment of acute promyelocytic leukemia: As2O3 induces NB4 cell apoptosis with downregulation of Bcl-2 expression and modulation of PML-RAR alpha/PML proteins. Blood. 1996;88(3):1052-1061.

17. Kim J, Lee JJ, Kim J, Gardner D, Beachy PA. Arsenic antagonizes the Hedgehog pathway by preventing ciliary accumulation and reducing stability of the Gli2 transcriptional effector. Proc Natl Acad Sci U SA 2010;107(30):13432-13437.

18. Tran PV, Lachke SA, Stottmann RW. Toward a systems-level understanding of the Hedgehog signaling pathway: defining the complex, robust, and fragile. Wiley Interdiscip Rev Syst Biol Med. 2013;5(1). 83-100.

19. Mazumdar T, DeVecchio J, Shi T, Jones J, Agyeman A, Houghton JA. Hedgehog signaling drives cellular survival in human colon carcinoma cells. Cancer Res. 2011;71(3):1092-1102.

20. Mazumdar T, DeVecchio J, Agyeman A, Shi T, Houghton JA. The GLI genes as the molecular switch in disrupting Hedgehog signaling in colon cancer. Oncotarget. 2011;2(8):638-645.

21. Hyman JM, Firestone AJ, Heine VM, et al. Small-molecule inhibitors reveal multiple strategies for Hedgehog pathway blockade. Proc Natl Acad Sci U S A. 2009;106(33):14132-14137.

22. Lauth M, Bergström A, Shimokawa T, Toftgård R. Inhibition of GLImediated transcription and tumor cell growth by small-molecule antagonists. Proc Natl Acad Sci U S A. 2007;104(20):8455-8460.
23. Williams JA, Guicherit OM, Zaharian BI, et al. Identification of a small molecule inhibitor of the hedgehog signaling pathway: effects on basal cell carcinoma-like lesions. Proc Natl Acad Sci U S A. 2003;100(8): 4616-4621.

24. Gulino A, Ferretti E, De Smaele E. Hedgehog signalling in colon cancer and stem cells. EMBO Mol Med. 2009;1(6-7):300-302.

25. Ji Z, Mei FC, Xie J, Cheng X. Oncogenic KRAS activates hedgehog signaling pathway in pancreatic cancer cells. J Biol Chem. 2007;282(19): 14048-14055.

26. Ju B, Spitsbergen J, Eden CJ, Taylor MR, Chen W. Co-activation of hedgehog and AKT pathways promote tumorigenesis in zebrafish. Mol Cancer. 2009;8:40.

27. Mizuarai S, Kawagishi A, Kotani H. Inhibition of p70S6K2 downregulates Hedgehog/GLI pathway in non-small cell lung cancer cell lines. Mol Cancer. 2009;8:44.

28. Riobo NA, Lu K, Ai X, Haines GM, Emerson CP Jr. Phosphoinositide 3-kinase and Akt are essential for Sonic Hedgehog signaling. Proc Nat Acad Sci U S A. 2006;103(12):4505-4510.

29. Perrot CY, Javelaud D, Mauviel A. Overlapping activities of TGF- $\beta$ and Hedgehog signaling in cancer: therapeutic targets for cancer treatment. Pharmacol Ther. 2013;137(2):183-199.

30. Schnidar H, Eberl M, Klingler S, et al. Epidermal growth factor receptor signaling synergizes with Hedgehog/GLI in oncogenic transformation via activation of the MEK/ERK/JUN pathway. Cancer Res. 2009;69(4):1284-1292.

31. Stecca B, Mas C, Clement V, et al. Melanomas require HEDGEHOG-GLI signaling regulated by interactions between GLI1 and the RAS-MEK AKT pathways. Proc Natl Acad Sci U S A. 2007;104(14):5895-5900.

32. Seto M, Ohta M, Asaoka Y, et al. Regulation of the hedgehog signaling by the mitogen-activated protein kinase cascade in gastric cancer. Mol Carcinog. 2009;48(8):703-712
OncoTargets and Therapy

\section{Publish your work in this journal}

OncoTargets and Therapy is an international, peer-reviewed, open access journal focusing on the pathological basis of all cancers, potential targets for therapy and treatment protocols employed to improve the management of cancer patients. The journal also focuses on the impact of management programs and new therapeutic agents and protocols on

\section{Dovepress}

patient perspectives such as quality of life, adherence and satisfaction. The manuscript management system is completely online and includes a very quick and fair peer-review system, which is all easy to use. Visit http://www.dovepress.com/testimonials.php to read real quotes from published authors. 